\title{
Prescrição de opioides em idosos com câncer: evidências sobre imunossupressão e seu impacto na prática clínica
}

\author{
Opioid prescription in elderly with cancer: evidence on immunosuppression and its impact \\ on clinical practice
}
Prescripción de opioides en ancianos con cáncer: evidencia sobre inmunosupresión y su impacto en la práctica clínica

Hugo Moura de Albuquerque Melo ${ }^{1 *}$, Jurema Telles de Oliveira Lima² ${ }^{2}$ Fabrício Oliveira Souto', José Luiz de Lima Filho'.

\section{RESUMO}

Objetivo: Conhecer, na população oncogeriátrica, a imunossupressão como efeito adverso do uso de opioides. Revisão Bibliográfica: Essa classe de drogas é utilizada para o controle de sintomas, principalmente, dor de intensidade moderada à forte. Há amplo conhecimento clínico sobre os efeitos colaterais mais comuns, como náusea, vômito e constipação, mas pouco é discutido sobre a imunomodulação provocada pelos opioides. Para a população de idosos com câncer, o controle de sintomas como a dor é fundamental no seguimento clínico. O adequado tratamento álgico é feito, muitas vezes, com a prescrição de opioides que entram para uso crônico e contínuo. Além das reações adversas clinicamente observadas, há vários relatos na literatura da investigação dos efeitos imunomoduladores dessas drogas e relação com risco aumentado de sepse e maior mortalidade. Considerações finais: Considerar a importância do efeito imunossupressor dos opioides no planejamento terapêutico, principalmente para os tratamentos contínuos, de forma a utilizar essa droga, sempre que bem indicada, mas com segurança.

Palavras-chave: Neoplasia, Envelhecimento, Analgésicos opioides, Imunossupressão.

\begin{abstract}
Objective: To know, in the oncogeriatric population, immunosuppression as an adverse effect of the use of opioids. Bibliographic Review: This class of drugs is used to control symptoms, mainly moderate to severe pain. There is ample clinical knowledge about the most common side effects, such as nausea, vomiting and constipation, but little is discussed about the immunomodulation caused by opioids. For the elderly population with cancer, the control of symptoms such as pain is essential in clinical follow-up. Adequate pain treatment is often done with the prescription of opioids that come in for chronic and continuous use. In addition to the clinically observed adverse reactions, there are several reports in the literature investigating the immunomodulatory effects of these drugs and their relationship with increased risk of sepsis and higher mortality. Final considerations: Consider the importance of the immunosuppressive effect of opioids in therapeutic planning, especially for continuous treatments, in order to use this drug, whenever well indicated, but safely.
\end{abstract}

Keywords: Neoplasm, Aging, Opioid analgesics, Immunosuppression.

\section{RESUMEN}

Objetivo: conocer, en la población oncogeriatrica, la inmunosupresión como efecto adverso del uso de opioides. Revisión bibliográfica: Esta clase de medicamentos se usa para controlar los síntomas, principalmente el dolor moderado a intenso. Existe un amplio conocimiento clínico sobre los efectos secundarios más comunes, como náuseas, vómitos y estreñimiento, pero se discute poco sobre la

\footnotetext{
1 Universidade Federal de Pernambuco (UFPE), Laboratório de Imunopatologia Keizo Asami (LIKA), Programa de Pósgraduação em Biologia Aplicada à Saúde (PPGBAS). Recife-Pernambuco. *E-mail: hugomouramelo@gmail.com

2 Instituto de Medicina Integral Professor Fernando Figueira, Serviço de Oncologia Clínica (IMIP). Recife-Pernambuco.
} 
inmunomodulación causada por los opioides. para la población anciana con cáncer, el control de síntomas como el dolor es esencial en el seguimiento clínico. El tratamiento adecuado del dolor a menudo se realiza con la prescripción de opioides que vienen para uso crónico y continuo. Además de las reacciones adversas observadas clínicamente, hay varios informes en la literatura que investigan los efectos inmunomoduladores de estos medicamentos y su relación con un mayor riesgo de sepsis y una mayor mortalidad. Consideraciones finales: Considere la importancia del efecto inmunosupresor de los opioides en la planificación terapéutica, especialmente para tratamientos continuos, para usar este medicamento, siempre que esté bien indicado, pero de manera segura.

Palabras clave: Neoplasia, Envejecimiento, Analgésicos opioides, Inmunosupresión.

\section{INTRODUÇÃO}

A população está envelhecendo e a proporção de categorias de faixa etária mais elevada aumentando. De acordo com a Organização Mundial da Saúde, é considerado idoso o habitante de país em desenvolvimento com 60 anos ou mais e em país desenvolvido o indivíduo com idade equivalente ou superior a 65 anos. $O$ envelhecimento é considerado um dos melhores indicadores da qualidade de qualquer sistema de saúde (FALANDRY C, et al., 2014).

A Organização das Nações Unidas projeta um ritmo de crescimento da população com 60 anos ou mais de $2,4 \%$ ao ano, antes de 2050 , e de $0,7 \%$ ao ano, entre 2050 e 2100 , resultando em um aumento de $50 \%$ dos idosos nas próximas quatro décadas nos países desenvolvidos, enquanto que, nos países em desenvolvimento, são estimadas taxas de crescimento anuais acima dos $3 \%$, proporcionando um aumento aproximado de 1,6 bilhões de idosos, em 2050 e 2,4 bilhões, em 2100 (UNITED NATIONS, 2013).

No Brasil as modificações ocorrem de forma radical e bastante acelerada. As projeções mais conservadoras indicam, em 2020, que o Brasil se torna o sexto país do mundo em número de idosos, com um contingente superior a 30 milhões de pessoas. Esse acelerado processo de envelhecimento populacional e o aumento na expectativa de vida chama atenção sobre as condições de saúde e morbidade dos idosos. Aliada a isso, a mudança no perfil demográfico brasileiro vem acompanhada por alterações no perfil epidemiológico, surgindo um novo paradigma de saúde, no qual as doenças infectocontagiosas são substituídas pelas doenças crônicas não transmissíveis, muitas vezes irreversíveis, como as neoplasias (AMARAL FLJ, et al., 2013).

As principais doenças crônicas não transmissíveis que afetam os idosos em todo o mundo são as neoplasias (câncer), doenças cardiovasculares (doença coronariana, hipertensão arterial sistêmica, acidente vascular encefálico), diabetes mellitus, doença pulmonar obstrutiva crônica, doenças musculoesqueléticas (artrite e osteoporose), doenças mentais (demência e depressão), diminuição da visão e cegueira (MOREIRA VG e LOURENÇO RA, 2013).

O câncer é um importante problema de saúde pública em países desenvolvidos e em desenvolvimento, sendo responsável por mais de 9,6 milhões de óbitos em 2018, com aproximadamente $70 \%$ em países de baixa e média renda, representando cerca de $15 \%$ de todas as causas de morte no mundo. À exceção do câncer de pele não melanoma, os tipos de câncer mais frequentes em homens são próstata $(31,7 \%)$, pulmão $(8,7 \%)$, intestino $(8,1 \%)$, estômago $(6,3 \%)$ e cavidade oral $(5,2 \%)$. Nas mulheres, os cânceres de mama $(29,5 \%)$, intestino $(9,4 \%)$, colo do útero $(8,1 \%)$, pulmão $(6,2 \%)$ e tireoide $(4,0 \%)$ figuram entre os principais (INCA, 2017).

Com isso, é crescente o desafio em conhecer e melhor cuidar dessa população, pois o avanço da idade está associado a um declínio progressivo na reserva funcional de múltiplos sistemas de órgãos e a uma maior incidência de doenças crônico degenerativas, como o câncer (HIGUERA O, et al., 2016).

Assim, conforme Pedersen JK, et al. (2016), idade é o fator de risco mais importante para desenvolver câncer, justificando a importância de estudos em relação a avaliação de pacientes oncogeriátricos. E nesta população de idosos com câncer, além da imunossupressão imputada pelo processo de envelhecimento e

REAS/EJCH | Vol.12(5) | e2868 | DOI: https://doi.org/10.25248/reas.e2868.2020 Página 2 de 8 
pela própria patologia neoplásica, este grupo de pacientes ainda vem sendo submetido a regulação da imunidade em decorrência do uso de opioides (VENTURA MT, et al., 2017; ZHANG X, et al., 2017).

O presente artigo teve como objetivo, então, apresentar uma revisão de literatura na qual sejam apresentados os efeitos adversos conhecidos e discutida a imunossupressão a partir do uso de opioides em idosos com câncer.

\section{REVISÃO DE LITERATURA}

\section{Envelhecimento populacional e oncogênese}

Nas últimas décadas, todas as regiões do mundo têm experimentado uma transição demográfica (ou seja, mudanças nos padrões de mortalidade, fertilidade e expectativa de vida) juntamente com mudanças epidemiológicas caracterizada pela importância emergente das doenças crônicas, como o câncer, doenças cardiovasculares, diabetes, dentre outras. (McKEOWN RE, 2009).

O aumento mundial da população idosa envolve um grande número de pessoas afetadas por essas doenças. Globalmente, as doenças não transmissíveis matam 38 milhões de pessoas a cada ano, e o câncer, de forma geral, é responsável por 22\% de todas as doenças (McKEOWN RE, 2009; POU SA, et al., 2017).

O fenômeno do envelhecimento demográfico está impulsionando a epidemia mundial dessas doenças crônicas como tendência social com amplo impacto na vida dos idosos. Demógrafos buscam explicações para a transição da mortalidade, colocando ênfase no papel da medicina moderna, ascensão nos padrões de vida e melhor nutrição, além de mudanças comportamentais e sociais (POU SA, et al., 2017).

Além disso, a chamada transição de saúde, focada nos determinantes socioculturais e comportamentais da saúde, interagem com a prestação de serviços de saúde e fornecem uma estrutura integrativa para que facilitem a identificação de fatores subjacentes, como as tendências de mortalidade (VALLS J, et al., 2009). O envelhecimento é, assim, um desafio para a saúde pública contemporânea, bem como um fator de risco para a oncogênese devido às alterações funcionais e fisiológicas próprias do envelhecer (POU SA, et al., 2017).

As neoplasias malignas provêm de alterações no processo apoptótico e/ou de proliferação celular gerando um desequilíbrio (heterostase), favorecendo acúmulos desordenados de células, que vão dar origem a um tumor capaz de gerar sinais e sintomas que se caracterizam como câncer. Com isso, disfunções nesses genes supressores que estão intimamente relacionados com a senescência celular podem aumentar a chance de mutações gênicas e induzir ao surgimento do câncer, uma vez que $50 \%$ dos cânceres humanos apresentam disfunção genética (KIM S, et al., 2002).

No Brasil, as neoplasias malignas foram responsáveis por 105.275 mortes, em 1990, e por 236.345 mortes, em 2015, correspondendo a $11,6 \%$ e a $17,4 \%$ do total estimado de óbitos, respectivamente. Para ambos os sexos, as taxas de mortalidade exibiram estabilidade (queda não expressiva) de 1990 para 2015. Entre as mulheres, o câncer de mama apresentou a maior taxa de mortalidade em 2015, seguido pelos cânceres de pulmão, cólon e reto, colo uterino e estômago. Já entre os homens, o principal foi o câncer de próstata, seguido pelos cânceres de pulmão, estômago, cólon e reto, e esôfago (GUERRA MR, et al., 2017).

Para a população de idosos com câncer, aos quais são atribuídos com razoável frequência diagnósticos já em condições avançadas de doença, o controle de sintomas como a dor é fundamental no seguimento clínico. O adequado tratamento álgico está então preconizado, para controle de dor moderada a grave com a prescrição de opioides, que entram na cesta medicamentosa para uso crônico e contínuo. Desse modo, os efeitos terapêuticos e adversos dessas drogas precisam ser amplamente conhecidos.

\section{Opioides}

Os opioides, no decorrer dos anos, já receberam várias nomenclaturas. Narcoanalgésicos, narcóticos, hipnoanalgésicos são alguns dos termos, hoje considerados impróprios por incluírem outras substâncias não pertencentes a classe dos derivados do ópio. Houve, ainda, a denominação genérica de opiáceos, sendo esta 
posteriormente restrita aos derivados naturais do ópio. Entretanto, houve evolução conceitual e o termo opioide passou a ser utilizado a todas as substâncias naturais, semissintéticas ou sintéticas que reagem com os receptores opioides, seja como antagonista ou agonista (GAITHER JR, et al., 2016).

Entre os opioides há poucas diferenças nos efeitos colateriais, sendo a maioria comum entre as drogas da classe. Na prática clínica são referidos com mais frequência, xerostomia, constipação, vômitos, nauseas e sedação. Em casos menos usuais, alterações cognitivas (ideias delirantes, alucinações), dispepsia, hiperalgesia, prurido, mioclonias e depressão respiratória (RIPAMONTI Cl, et al., 2012).

Os opioides podem atuar na reduão da peristalse com consequente maior permanência do bolo fecal no intestino. Com isso, facilita-se a constipação sendo consenso a necessidade da prescrição de laxantes associados aos opioides. Como orientação acessória estimula-se atividade física e aumento da hidratação (RIPAMONTI Cl, et al., 2012).

Já as náuseas e vômitos apresentam-se em maior intensidade nos primeiros dias de uso, com grande variação de intensidade entre indivíduos. Regularmente são indicados bromoprida, domperidona ou metoclopramida pelo efeito procinético e antidopaminérgico. $O$ haloperidol também pode ser utilizado, considerando-se a ação antagonista dopaminérgica (SMITH HS, 2009).

O efeito sedativo é mais usual bem no início da utilização da droga ou quando esta encontra-se em altas doses. Pode ter sua ação potencializada por outras medicações depressoras do sistema nervoso central. A maioria dos pacientes, entretanto desenvolve tolerância após algum tempo de tratamento e esse efeito é compensado, quando não influenciado por outros fatores (SMITH HS, 2009).

Nesse contexto, a tolerância a opioides pode surgir com a necessidade de realizar progressivos aumentos de dose para obtenção do efeito esperado, geralmente presente na utilização crônica da medicação. Entretanto, geralmente essa ascenção na dose encontra-se mais relacionada a piora de sintomas como a dor e não à tolerância farmacológica (RIPAMONTI Cl, et al., 2012).

A dependência física, quando identificada correlaciona-se a situações de abstinência em geral no em suspensões repentinas do medicamento, algo evitável com ajuste adequado da posologia e redução gradual (SMITH HS, 2009).

Dos efeitos adversos conhecidos, a depressão respiratória está entre os mais temidos. Conhecendo bem as drogas e prescrevendo com segurança percebe-se que esse efeito secundário não se desenvolve se 0 aumento da dose for gradual. Quando há necessidade de altas doses ou disfunções orgânicas, a faixa terapêutica fica comprometida sendo os efeitos colaterais mais facilmente observados. Clinicamente precedida por sonolência a depressão respiratória pode ser revertida com a suspensão da droga, redução da dose ou uso de antagonista opioide (naloxona) (SMITH HS, 2009).

Além dessas reações adversas clinicamente observadas, há vários relatos na literatura da investigação dos efeitos imunomoduladores dessas drogas. Contudo, as repercussões clínicas desses efeitos ainda precisam de maiores discussões, que possam resultar em orientações clínicas claras e preventivas.

\section{Opioides, suas ações imunorregulatórias e possíveis desfechos clínicos}

O uso e abuso de opioides têm sido associados à supressão do sistema imunológico no homem por mais de 100 anos. Já é bem aceito que eles modulam a função imune e há evidências crescentes que o uso de opioides no paciente cirúrgico e naqueles criticamente doentes tem um profundo efeito imunomodulador (FRIEDMAN H, et al., 2003). A regulação imune refere-se à interação entre células do sistema imunológico e mediadores e pode ser afetada por qualquer estímulo que prejudique a hematopoiese das células do sistema imunológico, desenvolvimento de suas componentes e mecanismos de feedback antígeno-anticorpo (ALHASHIMI M, et al., 2013).

Diferentes opioides afetam a função imune de maneira diferente, dependendo dos fatores relacionados às propriedades dos medicamentosos, dos fatores do hospedeiro e da duração da exposição (SACERDOTE P, 2006). Enquanto a inter-relação entre analgésicos opioides e o sistema imunológico não é simples e pode

REAS/EJCH | Vol.12(5) | e2868 | DOI: https://doi.org/10.25248/reas.e2868.2020 Página 4 de 8 
variar dependendo do opioide estudado, intervalo de dose da droga, espécies em que são testados, parâmetros imunológicos medidos e o curso do tempo do estudo, tem aumentado a disponibilidade de dados que indicam a imunossupressão no humano induzida por opioides em uma variedade de situações díspares (BUDD K e SHIPTON E, 2004).

À medida que as investigações sobre os opioides continuam, estudos anteriores relataram que essas drogas estão altamente associadas ao risco de efeitos imunossupressores, conforme revelado por estudos in vitro e in vivo (CHENG WF, et al., 2006). Os mecanismos subjacentes à imunossupressão induzida por opioides, embora não sejam bem delineados, podem incluir ações diretas e indiretas no sistema de imunidade celular (NGUYEN T, et al., 2009).

Alguns estudos sugerem que a morfina, a droga protótipo e mais estudada entre os opioides, tem efeitos nas imunidades inatas e adaptativas. O tratamento crônico com a morfina, in vivo, pode aumentar a diferenciação Th2 das células T CD4 +. Em contraste com a ativação dos linfócitos T CD4 + auxiliares, a atividade dos linfócitos T citotóxicos CD8 + é suprimida na resposta imune celular, quando a morfina é agudamente administrada (MOJADADI S, et al., 2009).

A morfina exerce influência sobre a imunidade do hospedeiro, alterando as respostas das células $T$ e $B$, atenuando a produção de anticorpos, reduzindo a atividade das células natural killers e suprimindo a proliferação e as funções dos linfócitos T e B. Além disso, a terapia opioide tende a reduzir o nível de linfócitos $\mathrm{T}(\mathrm{CD} 3+)$, incluindo subpopulações CD4 +, CD8 + e CD4 + / CD8 + duplamente positivas no sangue periférico de camundongos, o que, por sua vez, pode levar a uma redução de hipersensibilidade ao contato celular e respostas imunes humorais (VALLEJO R, et al., 2004). Desse modo, a supressão da imunidade do hospedeiro pela inibição das respostas das células $T$ pode ser um mecanismo de imunocomprometimento induzido por opioides (MING-CHENG C, et al., 2011).

Essa depleção do sistema imunológico geralmente pode resultar em infecção por germes oportunistas. A invasão de um hospedeiro por agentes infecciosos patogênicos inicia uma sequência de respostas imunes por meio de interações entre uma variedade de fatores de virulência e os mecanismos de vigilância imune do hospedeiro. Uma resposta imune inadequada pode não apenas resultar em falta de proteção, mas também contribuir para a doença (ROY S, et al., 2011).

O uso e abuso crônicos de opioides foram documentados não só no comprometimento do sistema imunológico, mas também no aumento do risco de infecção oportunista (ROY S, et al., 2006; DINDA A, et al., 2005). Isso é apoiado por estudos epidemiológicos que mostram maior prevalência de infecções oportunistas como tuberculose, infecção pelo HIV e pneumonia em viciados em opioides. São importantes sinalizações desse risco, apesar das inúmeras variáveis de confusão presentes nesses cenários. (NATH A, et al., 2002).

Em estudos com animais em que as variáveis podem ser controladas, o tratamento com morfina (usado como substituto da heroína) resulta em prejuízo imunológico significativo, com maior suscetibilidade a infecções oportunistas. A defesa contra microrganismos patogênicos é mediada pelas reações iniciais da imunidade inata e pela resposta posterior da imunidade adaptativa. Foi demonstrado que o abuso de morfina crônica afeta os dois braços da defesa imune (ROY S, et al, 2006; VALLEJO R, et al., 2004).

No entanto, vale reforçar que o câncer atua também com propriedades imunossupressoras. As duas principais populações de células $T$ que combatem as células cancerígenas são as células $T$ citotóxicas CD8 + e as células T auxiliares CD4 + tipo 1. No entanto, quando esses tipos de células se infiltram em um tumor, geralmente desenvolvem um estado exausto pelo qual são incapazes de proliferar e produzir moléculas efetoras (BAITSCH L, et al., 2011). Assim, as células T específicas do antígeno tumoral não conseguem restringir 0 crescimento tumoral. Existem muitas evidências que sugerem que o microambiente tumoral imunossupressor prejudica as funções tumoricidas da infiltração de células T citotóxicas CD8 + e células Th1, produzindo citocinas imunossupressoras e interferindo na aptidão metabólica das células T (WANG H, et al., 2017). 
Apesar de tudo isso, estudos atuais apontam para outras variáveis que também podem afetar a imunidade e ação ou metabolismo dos medicamentos administrados, como ativação do eixo hipotálamo-hipófise-adrenal e secreção de opiáceos endógenos e glicocorticoides, conhecidos por serem potencialmente imunossupressores (VALLEJO R, et al., 2004). Conforme observado por Filipczak-Bryniarsk I, et al. (2018), a sensação de dor e nocicepção está associada à ativação da reação inflamatória e, portanto, não é possível excluir a possibilidade de analgésicos agirem diferentemente nas células imunológicas ativadas e em repouso. Além disso, este estudo segue a afirmação de que a dor crônica na condição clínica ocorre como sintoma de doenças frequentemente associadas à desregulação da resposta imune, que podem ser exacerbadas pela terapia com opioides que afetam adversamente o sistema imunológico.

Dentre as evidências in vivo, vale ressaltar, entretanto, uma coorte retrospectiva de 50.658 pacientes com dor em uso de opioides, do Tennessee Medicaid, nos Estados Unidos. Houve associação no grupo em uso de opioides a altas taxas de mortalidade ambulatorial (115/ 10.000 pacientes-ano entre usuários de morfina) por outras causas que não a overdose (CHUNG CP, et al., 2018). Esse achado foi corroborado pelo estudo de Ray WA, et al. (2016), que mostrou um risco 1,64 vezes maior de mortalidade por todas as causas em pacientes em terapia crônica com opioide em comparação com aqueles que foram submetidos a analgesia com anticonvulsivantes ou baixa dose de antidepressivo.

Estudos cumulativos demonstraram que o tratamento com opioides pode estar associado a muitas outras consequências fisiopatológicas negativas, incluindo perda de homeostase e barreira intestinal, aumentando, dessa forma, o risco de sepse. Consistente com estudos in vitro, há uma análise recente sugerindo que pacientes sépticos tratados com opioides aumentam as taxas de mortalidade em comparação com aqueles não tratados com opioides (mortalidade em 28 dias de 10,35\% para pacientes tratados com opioides versus $2,4 \%$ para aqueles não tratados, com $p<0,001$ após ajuste para vários fatores de confusão) (ZHANG R, et al., 2018).

Sabe-se que entre esses pacientes, maior dor está correlacionada com menor qualidade de vida e que a dor, por si só, já está associada ao aumento da mortalidade (CHAU I, et al., 2018). Portanto, não há benefício em evitar opioides no contexto de dor moderada a intensa. Além disso, existe uma alta prevalência de dor em pacientes oncológicos e na população idosa, e o abandono da efetividade do controle da dor proporcionado pelo uso de opioides, sem substituição equivalente, é desumano e deletério, dado o efeito adverso muito significativo da dor e do estresse na progressão do câncer (WIGMORE T, et al., 2016).

\section{CONSIDERAÇÕES FINAIS}

Esta revisão propõe-se, então, a elucidar esse viés imunossupressor, pouco discutido na prática clínica, dentre os demais efeitos colaterais do uso de opioides, para que seja considerado no momento de iniciar tratamentos com expectativa de cronicidade. Com isso, não se quer destituir a importância dos opioides, mas sim estimular a reflexão sobre o acompanhamento estrito dos efeitos adversos clinicamente apresentados avaliando o risco/benefício da terapia proposta, mas sempre consciente de que o objetivo principal a ser alcançado é a qualidade de vida do paciente.

\section{AGRADECIMENTOS E FINANCIAMENTO}

Agradecemos ao Laboratório de Imunopatologia Keizo Asami da UFPE e ao Serviço de Oncogeriatria do IMIP, por possibilitarem o estudo e aprofundamento nesta temática.

\section{REFERÊNCIAS}

1. AL-HASHIMI M, et al. Opioids and immune modulation: more questions than answers. British Journal of Anesthesia, 2013; 111(1): 80-88.

2. AMARAL FLJ, et al. Apoio social e síndrome da fragilidade em idosos residentes na comunidade. Ciência e Saúde Coletiva, 2013; 18(5): 1835-1846. 
3. BAITSCH L, et al. Exhaustion of tumor specific CD8(+) T cells in metastases from melanoma patients. J. Clin. Invest, 2011; 121(6): 2350-2360.

4. BUDD K, SHIPTON E. Acute pain the immune system and opioimmunosuppression. Acute Pain, 2004; 6(3-4): 123135.

5. CHENG WF, et al. Chimeric DNA vaccine reverses morphine-induced immunosuppression and tumorigenesis. Mol Ther, 2006; 13(1): 203e10.

6. DINDA A, et al. Immunomodulatory effect of morphine: therapeutic implications. Expert Opin Drug Saf, 2005; 4: 669675.

7. FALANDRY C, et al. Biology of Cancer and Aging: A Complex Association With Cellular Senescence. Journal Of Clinical Oncology, Alexandria, 2014; 32(24): 2604-2610.

8. FILIPCZAK-BRYNIARSK I, et al. In contrast to morphine, buprenorphine enhances macrophage-induced humoral immunity and, as oxycodone, slightly suppresses the effector phase of cell-mediated immune response in mice. International Immunopharmacology, 2018; 54: 344-353.

9. FRIEDMAN H, et al. Microbial Infections, Immunomodulation, and Drugs of Abuse. Clinical Microbiology Reviews, 2003; 16(2): 209-219.

10. GAITHER JR, et al. The Association Between Receipt of Guideline-Concordant Long-Term Opioid Therapy and AllCause Mortality. Journal Of General Internal Medicine, 2016; 31(5): 492-501.

11. GALVÃO TF, PANSANI TSA, HARRAD D. Principais itens para relatar Revisões sistemáticas e Meta-análises: A recomendação PRISMA. Epidemiologia e Serviços de Saúde, 2015; 24(2), 335-342.

12. GUERRA MR, et al. Magnitude e variação da carga da mortalidade por câncer no Brasil e Unidades da Federação, 1990 e 2015. Rev. bras. epidemiol., São Paulo, 2017; 20(Suppl. 1), 102-115.

13. CHAU I, et al. Association of quality of life with disease characteristics and treatment outcomes in patients with advanced gastric cancer: Exploratory analysis od RAINBOW and REGARD phase III trials. Eur J Cancer 2018; 107:115-123.

14. CHUNG CP, et al. Comparative out-of-hospital mortality of long-acting opioids prescribed for non-cancer pain: $A$ retrospective cohort study. Pharmacoepidemiol Drug Saf. 2019;28(1):48-53.

15. HIGUERA O, et al. Management of pancreatic cancer in the elderly. World Journal Of Gastroenterology, Pleasanton, 2016; 22(2): 764-775.

16. INSTITUTO NACIONAL DO CÂNCER JOSÉ ALENCAR GOMES DA SILVA (INCA). Estimativa 2018: Incidência de Cancer no Brasil. Rio de Janeiro: INCA, v. 64, n. 1, p. 199-120, 2017.

17. KIM S, et al. Telomeres, aging and cancer: In search of a happy ending. Oncogene, 2002; 21(4): 503-511.

18. MCKEOWN RE. The Epidemiologic Transition: Changing Patterns of Mortality and Population Dynamics. American Journal of Lifestyle Medicine, 2009; 3(1): 19-26.

19. MING-CHENG C, et al. Influence of morphine on host immunity. Acta Anaesthesiologica Taiwanica, 2011; 49(3): 105108.

20. MOJADADI S, et al. Acute morphine administration reduces cell-mediated immunity and induces reactivation of latent herpes simplex virus type 1 in BALB/c mice. Cell Mol Immunol, 2009; 6(2): 111e6.

21. MOREIRA VG, LOURENÇO RA. Prevalence and factors associated with frailty in an older population from the city of Rio de Janeiro, Brazil: the FIBRA-RJ Study. Clinics (São Paulo, Brazil), 2013; 68(7): 979-985.

22. NATH A, et al. Molecular basis for interactions of HIV and drugs of abuse. J Acquir Immune Defic Syndr, 2002; 31: $62-9$.

23. NGUYEN T, et al. Citalopram enhances B cell numbers in a murine model of morphine-induced immunosuppression. Pain Pract., 2009; 9(3): 195-205.

24. PEDERSEN JK, et al. Cancer and aging: Epidemiology and methodological challenges. Acta Oncologica, London, 2016; 55(1): 7-12.

25. POU SA, et al. Burden of cancer mortality and differences attributable to demographic aging and risk factors in Argentina, 1986-2011. Cad. Saúde Pública, Rio de Janeiro, 2017; 33(2): e00016616.

26. QUAGLIO G, et al. Prevalence of tuberculosis infection and comparison of multiple-puncture liquid tuberculin test and Mantoux test among drug users. Scand J Infect Dis, 2002; 34: 574-576.

27. RIPAMONTI C, et al. Management of cancer pain: ESMO Clinical Practice Guidelines. Annals Of Oncology, 2012; 23(7): 139-154.

28. RAY WA, et al. Prescription of long-acting opioids and mortality in patients with chronic non-cancer pain. JAMA 2016;315(22):2115-2423.

29. ROY S, et al. Modulation of immune function by morphine: implications for susceptibility to infection. J Neuroimmune Pharmacol. 2006; 1:77-89.

30. ROY S, et al. Opioid drug abuse and modulation of immune function: consequences in the susceptibility to opportunistic infections. J. Neuroimmune, 2011; 6(4): 442-65.

31. SACERDOTE, P. Opioids and the immune system. Palliative Medicine, 2006; 20(8): 9-15.

32. SMITH HS. Opioid Metabolism. Mayo Clinic Proceedings, 2009; 84(7): 613-624.

33. UNITED NATIONS, Department of Economic and Social Affairs, Population Division. World Population Prospects: The 2012 Revision, Highlights and Advance Tables. Working Paper No. ESA/P/WP.228, 2013, 118p.

34. VALLEJO R, et al. Opioid therapy and immunosuppression. Am J Ther, 2004; 11: 354-365.

35. VALLS J, et al. RiskDiff: a web tool for the analysis of the difference due to risk and demographic factors for incidence or mortality data. BMC Public Health, 2009; 9(1): 473. 
36. VENTURA MT, et al. Immunosenescence in aging: between immune cells depletion and cytokines upregulation. Clinical and Molecular Allergy, 2017; 15(1).

37. WANG H, et al. Metabolic Regulation of Tregs in Cancer. Opportunities for Immunotherapy. 2017; 8(3): $583-592$.

38. WIGMORE T, et al. Opioids and cancer: friend or foe? Curr Opin Support Palliat Care 2016;10(9):109-118.

39. ZHANG R, et al. Prescription opioids are associated with higher mortality in patients diagnosed with sepsis: A retrospective cohort study using electronic health records. PLoS ONE, 2018;13(1): e0190362.

40. ZHANG X, et al. The Biology of Aging and Cancer. The Cancer Journal, 2017; 23(4): 201-205. 